\title{
Basic research on lattice structures focused on the reliance of the cross sectional area and additional coatings
}

\author{
Julia Kessler ${ }^{1, *}$, Nicolae Bâlc ${ }^{2}$, Andreas Gebhardt ${ }^{1}$, and Karim Abbas ${ }^{1}$ \\ ${ }^{1}$ Aachen University of Applied Sciences, Faculty of Mechanical Engineering and Mechatronics, \\ 52064 Goethestr 1, Aachen, Germany \\ ${ }^{2}$ Technical University of Cluj-Napoca, Department of Manufacturing Engineering, 400641 B-dul \\ Muncii 103-105, Cluj-Napoca, Romania
}

\begin{abstract}
This scientific survey is about periodic lattice structures which are made by Selective Laser Melting (SLM). Selective laser melting is based on Additive Manufacturing. The increased use and increasing demand of lattice structures in different fields of applications forms the necessity of a closer look on complex structures. Lattice structures can be found in different fields of applications for example in lightweight applications, filters and heat exchangers. Because of the expanding of application areas and thus arising requirements, the quality improvement is indispensable. Additive manufacturing and especially the SLM process enable the manufacturing of highly complex shapes and structures. Further it allows the integration of lightweight structures within to be manufactured applications. These high performance structures and applications need specific boundary and process conditions [1-3]. The main aim of this survey is an extraction of important parameters concerning the shape of lattices. A first focus will be on mechanical properties and the therefore necessary tensile tests.
\end{abstract}

\section{Introduction}

Selective Laser Melting is a technology that is based on Additive Manufacturing. It can be divided into three process steps, the pre-process, main process and post-process. During the main process the product is printed. Therefore a metal powder is spread onto a building platform and then an outline is locally melted with a laser. On top of this layer a new layer of metal powder is applied followed by a laser melting step. This process is repeated until the whole shape is generated. The shape is defined by a CAD data. Almost no limit can be set on the complexity of shapes, what makes this kind of manufacturing technology unique compared to conventional technologies. Cavities or filigree structures like lattice structures can be generated.

There is a great potential in lattice structures like material and time saving and improving the mechanical properties of products. To exploit the maximum potential it is

${ }^{*}$ Corresponding author: kessler@fh-aachen.de 
necessary to take a closer look at the process flow and shape properties of lattice structures 3-5].

Several researches already carried out a great variety of mechanical and microstructural test focusing on the properties of lattice structures. These kinds of investigations allow a fundamental understanding and the comparison of result. P. Li et al. for example analysed the deformation behaviour of lattice structures and used a reduced initial system to get the required data for FE analysis. For this purpose uniaxial tension tests were performed and the FE analysis adjusted. A comparison of a real uniaxial compression test and a prediction via $\mathrm{FE}$ analysis showed similar results. For more complex structures the prediction still showed disagreements [6].

A further focus was investigated by S. McKown et al. They dealt with the dynamic loading test on lattice structures. Therefore several unit cell types were tested with compression tests and blast tests. The conclusion extracted out of the survey shows that within dynamic loading, depending on the set parameters, the compression strength increases with the loading rate. Comparing the blast loading and quasi-static loading the deformation mechanism is similar [7].

The above-mentioned investigations are based on periodic lattice structures. Else to that Chunze Yan et al. took an interest in complex Schoen gyroid structures which as well are periodic with the difference that the structures are designed by circular struts and spherical cores instead of regular struts. Compression tests on this type of structures proved that there is an anisotropic behaviour depends on the orientation of the lattice structure. In context to that fact the mechanical properties are direction depended $[8,9]$.

These introduced investigations on different lattice structures and different types of unit cells should show the great potential and possible uses of those high performance structures.

\section{Experimental set - up}

The following study is aiming at the determination of a great range of impacts affecting 3D generated lattice structures as well as to link dependencies. The base for this survey is a preliminary study which is going to be expanded with different specimens and evaluation methods. In this preliminary study three different square tensile specimens $(8 \times 8 \times 100 \mathrm{~mm})$ were generated, using $316 \mathrm{~L}$ stainless steel with an average grain size of $30-60 \mu \mathrm{m}$. Tests were carried out on a massive specimen, a lattice structure and a coated lattice structure. First tensile test opened up a great discrepancy within the tensile strength, which raised some new issues. Out of this survey new focuses were extracted. A particularly conspicuous aspect is the great reached tensile strength of the lattice structure. Below the experimental set up is described [10].

The Selective Laser Melting machine used to generate the specimens is the M1 Cusing which works with a $400 \mathrm{~W}$ fiber laser. As above described the tests are based on $316 \mathrm{~L}$ stainless steel. In order to guarantee the same product quality and properties all specimens are built with the same process parameters. The scanning speed is set to $1450 \mathrm{~mm} / \mathrm{s}$ for outlines and $1000 \mathrm{~mm} / \mathrm{s}$ for surface exposure with the constant laser power of $180 \mathrm{~W}$. Regarding the exposure strategy the hatch distance is set to $80 \mu \mathrm{m}$. These adjustments have been extracted as the process optimum and will be no further discussed within this survey.

A series of different specimens and tensile test which are tested on a "Zwick" experimental machine, are the basis for this study. The range of samples covers different lattice structures, coated lattice structures, vertical struts and different rods with a similar cross sectional area. For each type four tensile specimens are printed. One specimen is intended as a reference to calibrate the tensile machine. The square rod shape is adjusted to the tensile machine and is not based on a DIN standard. Table 1 shows the four main 
groups and different sections of the specimens and Table 2 shows the main characteristics of the different specimens and their unit cells.

Table 1.Sections of the specimens for the experimental set up.

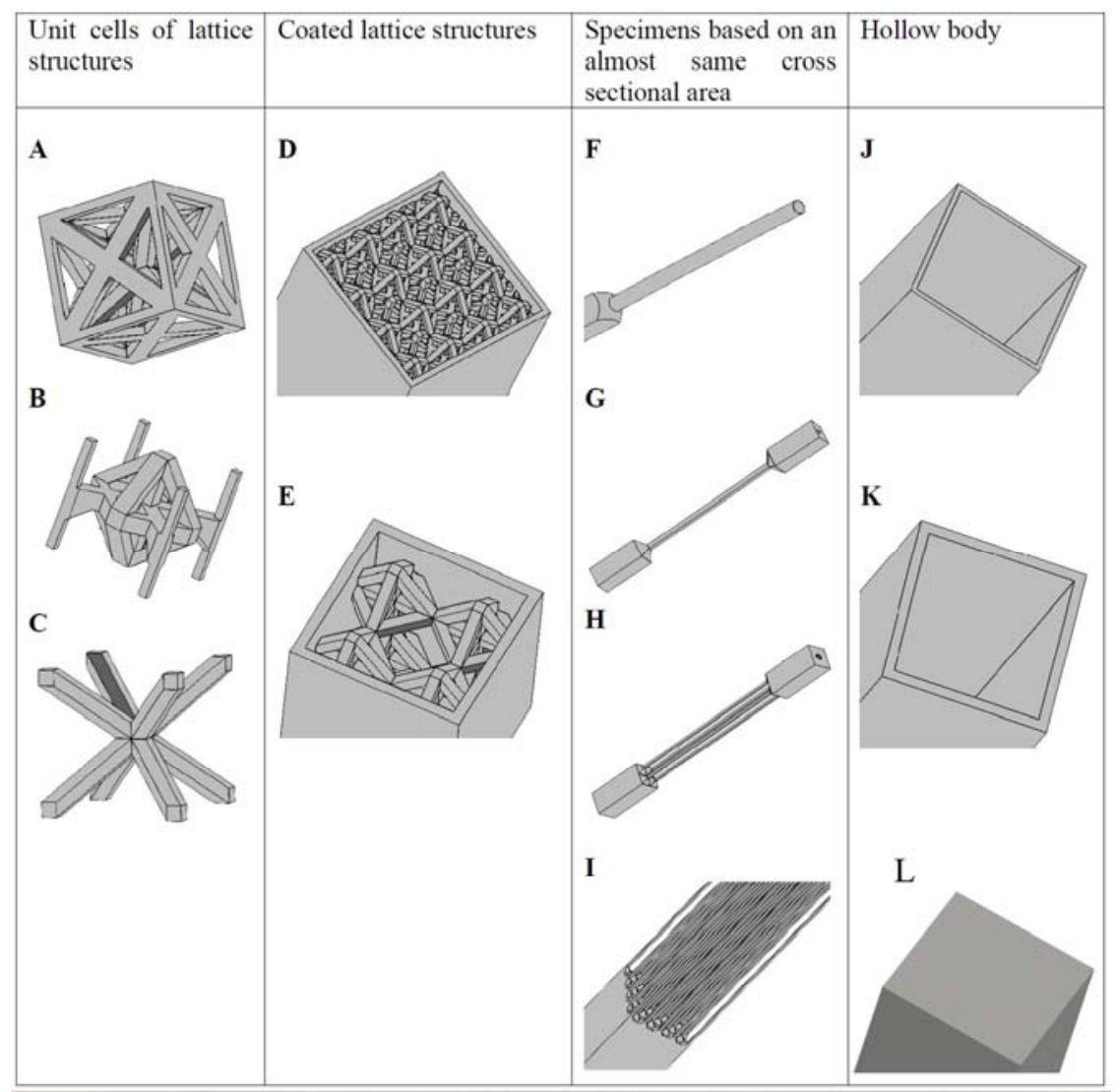

Table 2. Properties of the specimens.

\begin{tabular}{|l|l|l|l|l|l|l|}
\hline No. & $\begin{array}{l}\text { Specimen and type } \\
\text { of the unit cell }\end{array}$ & $\begin{array}{c}\text { Coating } \\
(\mathbf{m m})\end{array}$ & $\begin{array}{c}\text { Strut size } \\
\mathbf{( m m})\end{array}$ & $\begin{array}{c}\text { Cell size } \\
\mathbf{( m m})\end{array}$ & $\begin{array}{c}\text { Vertical } \\
\text { struts }\end{array}$ & $\begin{array}{c}\text { Smallest cross } \\
\mathbf{s e c t i o n a l} \\
\left(\mathbf{m m}^{2}\right)\end{array}$ \\
\hline A & Truss structure & - & 0.3 & 2 & yes & 8.393 \\
\hline B & Octahedral & - & 0.3 & 2 & yes & 3.69 \\
\hline C & Truss structure & - & 0.3 & 2 & - & 2.88 \\
\hline D & Coated octahedral & 0.3 & 0.3 & 2 & yes & 11.49 \\
\hline E & Coated octahedral & 0.5 & 0.5 & 4 & - & 16 \\
\hline F & Ring face & - & - & - & - & 3.675 \\
\hline G & Square & - & - & - & - & 3.686 \\
\hline H & Split square 4x & - & - & - & - & 3.688 \\
\hline I & Split square 41x & - & - & - & - & 3.69 \\
\hline J & Hollow body & 0.3 & - & - & - & 9.24 \\
\hline K & Hollow body & 0.5 & - & - & - & 15 \\
\hline L & Massive & - & - & - & - & 64 \\
\hline
\end{tabular}




\section{Results and discussions}

The Table 3 below shows the average values of the second series of test. Within the table reached tensile strength $\mathrm{Rm}$ of the different specimens is listed, these become accessible with the ratio of the highest reached force $F_{m}$ and the base area $A$. For the required calculation the base area of the specimens was used and which has a value of $64 \mathrm{~mm}^{2}$. It is not the strained cross sectional area that is integrated to the calculation. In this case it should be noted that the calculated results represent a mixture of component test and material science. Hence the table includes the smallest strained cross sectional area that turned out to be the breaking point of the specimens and further the main major weakness. Together with those values the actual tensile strength is calculated and listed in the column $\mathrm{R}_{\mathrm{m} \text {-theo. }}$. These calculated values finally show the significances and deviations. The results are in a descending order of the tensile strength. Like above mentioned the focus of this experimental set up are several structural properties including a range of different lattice structures. The complexity of this lattice structures is defined by their diversity of unit cell structures. Because of the fact that lattices structures are integrated within products a further, to test, aspect is the coating of lattice structures. Here the effect of a coating is evaluated. In order to interpret affects and to make conclusions out of the results, the experimental set up is further expanded with hollow body specimens. These specimens represent the coatings of the bevor tested coated lattice structures. The interaction of a coating and structure should be uncovered. A final focus is on the cross sectional area of the specimen B listed in Table 2. The great achieved tensile strength of almost $1172 \mathrm{MPa}$ is a significant value that needs a special attention. Therefore the dependence and relation of the cross sectional area is tested.

Thus a variety of different specimens with almost the same cross sectional area are included in the experimental set up. Besides the cross sectional area different geometries and different distribution of the cross section are the main aspects that are looked at.

Taking a look at the Table 3, four rough divisions can be separated. Firstly the specimens that show comparable results to the reference massive probe $\mathrm{L}(\mathrm{E})$, and the once that have slight $(\mathrm{G}, \mathrm{I}, \mathrm{F}, \mathrm{H}, \mathrm{C})$, great $(\mathrm{D}, \mathrm{K}, \mathrm{A})$ and significant $(\mathrm{B}, \mathrm{J})$ deviations to the reference probe. The green marked specimens are on the one side the massive probe itself and a lattice structure $\mathrm{E}$ with a coating. The lattice structure has a unit cell with the size of $4 \times 4 \times 4 \mathrm{~mm}$, a strut size of $0.5 \times 0.5 \mathrm{~mm}$ and a $0.5 \mathrm{~mm}$ coating. Both the highest reached force and theoretical cross section are a quarter of the massive specimen. Taking these values for a calculation of the theoretical tensile strength, the result is a nearly similar value as the reference. Firstly this might be an indication that the tensile strength is based on the cross section of the specimen. Continuing with the next group of probes these results are ranged in those with great deviations.

The specimen $\mathrm{D}$, which is a coated lattice structure with an octahedral geometry, and the specimen A, with a truss lattice structure, have almost the same cross sectional area of the major weakness with an average deviation. Their theoretical tensile strength is comparable. Deviating from these two structures the specimen $\mathrm{K}$ is matched into this group because of its reached similar values of the component test. Interesting about this is the fact, that the theoretical cross section has an alike as E, but a lower theoretical calculated strength. Hence, there is further the necessity to rethink the theory of the behaviour of the cross section and the relation to the tensile strength in case of lattice structures. Further on the following specimens, marked in red, also show great deviations compared to the others. Above all the specimen B, which is a lattice structure including octahedral unit cells with further vertical struts, has an unexpected reached theoretical value of its tensile strength. This sample is the one that is the fundament for the further design of the experimental setup. Conspicuous about the second specimen $\mathrm{J}$ belonging to this group is that its reached 
values are significantly lower than the once described above $(\mathrm{D}+\mathrm{A})$. With a mostly alike cross section compared to D and A, just and almost half as force is achieved. Even lower strain is reached compared to the specimen $\mathrm{B}$, which has an almost 2.5time smaller cross section. The shape of the last division is based on the results of the deviations of the above discussed lattice structure B. Almost every specimen has the same cross section with the difference of the chosen design and distribution.

The aim was to find out if specimens with the same cross section show the same significant deviations as the lattice structure. All in all the results show that having the same cross section does not affect the tensile strength.

Table 3. Results of the tensile test.

\begin{tabular}{|l|l|l|l|l|}
\hline Specimen No. & Rm [MPa] & Fm $[\mathbf{k N}]$ & Atheo $\left[\mathbf{m m}^{2}\right]$ & Rm-theo [MPa] \\
\hline L & 621 & 39.75 & 64 & 621.09375 \\
\hline E & 150 & 9.95 & 16 & 621.875 \\
\hline D & 134 & 8.57 & 11.49 & 745.86597 \\
\hline K & 131 & 8.37 & 15 & 558 \\
\hline A & 110 & 7.05 & 8.393 & 839.985702 \\
\hline B & 67.5 & 4.325 & 3.69 & 1172.08672 \\
\hline J & 58 & 3.72 & 9.24 & 402.597403 \\
\hline G & 33 & 2.1 & 3.686 & 569.723277 \\
\hline I & 29 & 1.87 & 3.69 & 506.775068 \\
\hline F & 26.75 & 1.71 & 3.675 & 465.306122 \\
\hline H & 25 & 1.59 & 3.688 & 431.127983 \\
\hline C & 22.5 & 1.45 & 2.88 & 503.472222 \\
\hline
\end{tabular}

\subsection{Curve diagram}

Taking a look at the curve diagram shown in Fig.1, a few values should be described and discussed. The curves show regular behaviour with a continuous transition from the elastic to the plastic area and the presence of Hook's law [11].Nevertheless, two specimens show extraordinary progressions. Beginning with the specimen $\mathrm{E}$ that has the greatest tensile test of this series, it is noticeable that there are two peaks. A high peak followed by a lower peak. It is difficult to interpret the appearance and reasoning of the second peak. A possible reason could be an early strip off of a layer and a geometry structure, which causes a temporary drop. This means that the strip off is internal, however the coat is not affected. This fact might cause a further shortly increasing tensile strength until the total break appears. On the other hand there is a specimen (C) that shows extraordinary strain behaviour. It has the greatest strain measured within this experimental set up. The cell type of this specimen is an octahedral, without any vertical struts. All in all this leads to a similar strain behaviour and great deformation of the specimen. 


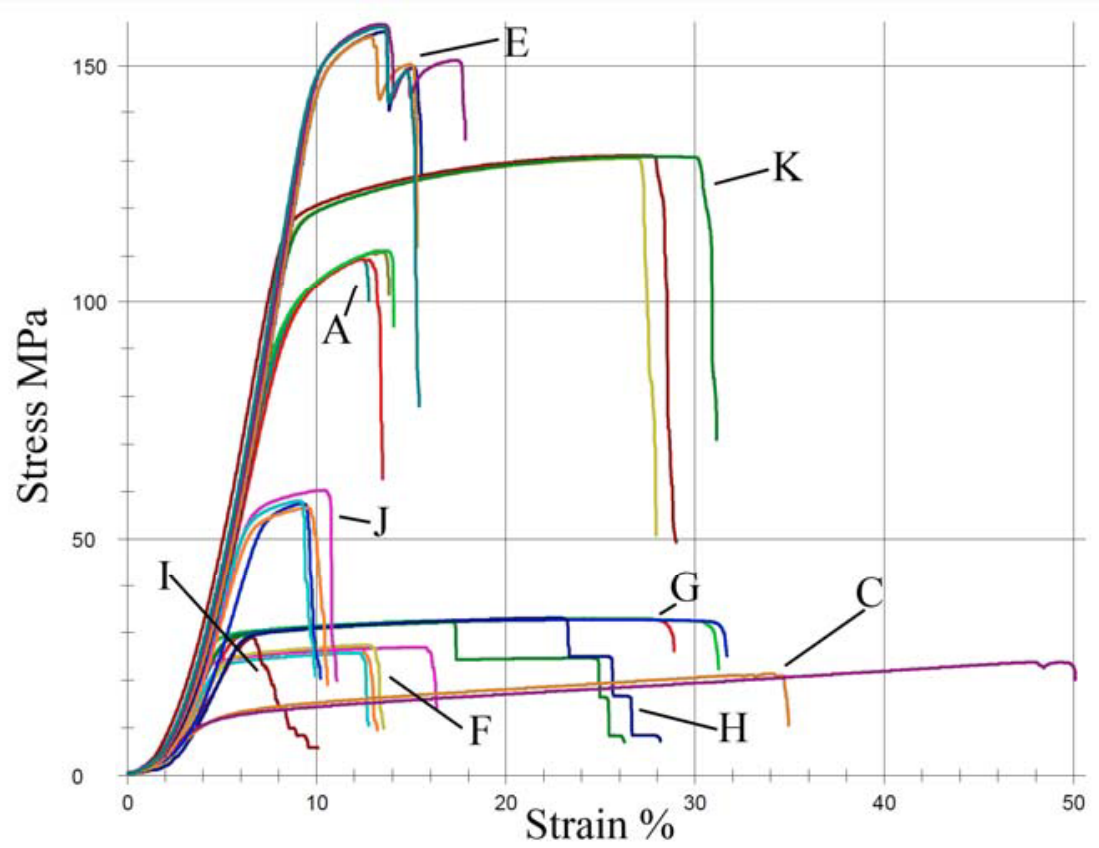

Fig. 1. Curve graphic of several tensile tests regarding this investigation.

\section{Conclusions}

The above mentioned results enable new starting points to verify theories regarding the properties of lattice structures. Actually the aim was to find new interactions of different factors that affect the lattice structures. In carrying out the investigation several results were extracted that need a new and adjusted test series as well as a closer look on it. A special abnormality was discovered at the specimen E. As already told there is an interesting curve progression of the tensile test. Because of the fact that reached tensile strength is similar to the tensile strength of the specimen L, it seems that neither the cell structure nor the coating has an effect on the tensile strength.

This fact is the reason for a new theory. It is necessary to have a closer look on the dimensioning of the unit cell and the coating itself. Perhaps the great cell structure opens up the possibility for a local constriction which leads to a predetermined breaking point. Moreover the comparison of the specimens B and D introduces new issues. Except for the surrounding coat of $\mathrm{D}$ the specimens are identical nevertheless the reached theoretical tensile strength of sample D is lower compared to B. One of the main theories was that a major weakness is at the lowest cross sectional area. Caused by the SLM process itself, generated parts show anisotropic behaviour $[12,13]$.

Concerning the specimen B it was necessary to reduce the structure to a specimen with just the same cross sectional area. This reduction and comparison showed that a reduction is not achieving the same results just because of having the same cross sectional area.

Regarding to this facts it is still impossible to make more concrete conclusions. There are still several parameters that might influence the gained achievements. These could be the shape, the microstructure or process parameters, which are not yet taken into consideration. Even slight deviations are an error source for calculations and misinterpretation. All these issues need to be reduces or eliminated in further studies [14]. 
This paper was supported by the project entitled "Boosting the scientific excellence and innovation capacity in Additive Manufacturing of the Technical University of Cluj-Napoca" (AMaTUC), founded by the European Union's Horizon 2020 research and innovation programme under grant agreement No. 691787 (www.amatuc.com).

\section{References}

1. J. Glasschröder, Applied Research at the Laboratory for Additive Manufacturing (iwb Application Centre Augsburg, 2013)

2. R. Poprawe, Management Circle 3, 14-50 (2015)

3. A. Gebhardt, Understanding Additive Manufacturing (Carl Hanser Verlag, Munich, 2011)

4. Fraunhofer ILT, Annual Report 2015, http://www.ilt.fraunhofer.de/content/dam/ilt/de/ documents/Jahresberichte/JB13/H_JB13_75.pdf, accessed 2016

5. C. Hamm, Evolution of Lightweight Structures - Analysis and Technical Applications (Springer Publications, 2015)

6. P. Li, Z. Wang, N. Petrinic, C.R. Siviour, Mater.Sci.\& Eng. A 614, 116 (2014)

7. S. McKown, Y. Shen, W.K. Brookes, C.J. Sutcliffe, W.J. Cantwell, G.S. Langdon, G.N. Nurick, J SANDW STRUCT MATER 13, 479-501 (2011)

8. C. Yan, L. Hao, A. Hussein, P. Young,D. Raymont, Materials \& Design 52, 638-647 (2014)

9. A. Hussein, C.Yan, P. Young, L. Hao, Rtejournal 12, 3 (2015)

10. J. Kessler, N. Balc, A. Gebhardt, K. Abbas, AMM 808, 193-198 (2015)

11. H. Burkhard, Material Testing-Determination of Material Properties (Carl Hanser Verlag, Munich, 2003)

12. A. Schwarz, Joining of Generatively Manufacutred Parts Made from1.4404 (Master Thesis, 2015)

13. D. Ziebura, Development of a Systematology for Optimal Parameter Finding in the SLM-Process on the Example of IN 625 (Master Thesis, 2015)

14. J. Kessler, N. Balc, A. Gebhardt, K. Abbas, Physics Procedia 83, 833-838 (2016) 